\title{
Mathematical Methods for Dissolved Phosphorus Concentrations
}

\author{
Kuiyuan Li and Josaphat Uvah \\ Department of Mathematics and Statistics, University of West Florida, Pensacola, FL 32514 \\ Email: kli@uwf .edu
}

\begin{abstract}
Phosphorus is a nutrient contained in fertilizer that can run off from lawns and crops. Predicting the phosphorus concentrations is an important component in determining how phosphorus is cycled in the surrounding ecosystem. In this paper, we present the development of two mathematical methods. The first is a steady-state diffusion ordinary differential equation with given initial data. For this method, we estimate the unknown parameter values using least squares approximations for the data set without the boundary values. The second method is identical to the first except that the boundary values are imposed. We then distinguish our methods from existing approaches by deploying homotopy continuation to connect all time stages. With this approach, phosphorus concentrations can be estimated at all times and any depth. Using real-life data, we give an example to show that the methods are not only easy to use, but also provide estimates between any time stages and at any depth.
\end{abstract}

Keywords: Steady-state diffusion, time stages, homotopy continuation.

\section{Introduction}

Diagenesis refers to the sum of processes by which sediments of matter undergo changes from deposition to solidication. Together with the broader field of biogeochemistry, diagenesis encompasses the subject areas of biology, physics and chemistry [1]. The mathematical modeling of diagenesis helps scientists better understand nutrient cycling and predict future nutrient conditions. Thus, the diagenesis modeling of organic matter and nutrients continues to be of interest to biogeochemical researchers [2,3]. In particular, modeling phosphorus diagenesis is important in the United States because communities, environmentalists and governmental agencies utilize it to determine overall phosphorus budgets and cycling for the ecosystem. Indeed, the negative effects of phosphate, the main form of dissolved inorganic phosphorus, on water quality are well-known [4]. For consumers, environmentalists and legislators, these known negative effects of phosphorus on water quality require accurate estimates of phosphorus concentrations in water and soil.

In recent years, diagenetic models coupled with hydrodynamic models have been used to estimate phosphorus concentrations in overlaying water [4]. Established diagenetic models are not in short supply. However, these have two related limitations: (a) most of the models are rather complex and intractable; and (b) due to the first issue, researchers resort to steady-state models (independent of time) which do not account for early stages of diagenesis. Consequently, these models have little practical use to biogeochemists who are interested in various stages of the evolution [5]. A third major issue arises from the cost and difficulty of collecting samples frequently. Existing models often provide estimates at discrete time stages $t_{1}, t_{2}, t_{3}, \ldots, t_{n}$, where any two adjacent time stages are distant from each other. For each time stage at a fixed location, samples are collected at various depths. A model utilizes this data to estimate concentration levels for any depth at that time stage. Conspicuously missing is a means to predict concentration levels at times other than those time stages. It is, therefore, essential to develop user-friendly, time-dependent methods, to estimate concentration values between sampling dates.

In this paper, we use two related models: (a) the steady-state diagenetic diffusion model, an ordinary differential equation with given data, and (b) the diagenetic model with boundary conditions. In the first method, the solution contains unknown parameters which we estimate by least squares approximation. For the second method, the boundary conditions suffice to obtain parameter values. Furthermore, we utilize the well-known homotopy continuation method $[6,7]$ to connect the time stages so as to provide concentration estimates at any time and any depth. Homotopy continuation produces smooth curves that 
bridge the gap between two points with known data. In addition, we establish a numerical algorithm for implementing the methods with homotopy continuation. In order to show how all this works in practice, we give an example using real data collected in a bay near the Gulf of Mexico.

\section{Mathematical Methods}

We consider a permeable box in which dissolved phosphorus enters by means of diffusion and advection. Sources and sinks constitute the reactions that also affect the phosphorus concentration in the box.

Let $\vec{F}_{D}=$ diffusive flux,

$C=$ phosphorus concentration,

$D=$ diffusion constant,

$\vec{v}=$ advection vector,

and $R=$ reaction,

then the steady-state diffusive flux is

$$
\vec{F}_{D}=-D \nabla C
$$

and the general diagenetic equation is

$$
\frac{\partial C}{\partial t}=-\operatorname{div} \vec{F}_{D}-\operatorname{div}(\vec{v} C)+\sum R
$$

The diffusive constant depends on temperature. The porosity of sediment is measured as the volume of porewater in the box divided by the total volume. The sediment also affects the path of the porewater flow. This effect is called tortuosity. Measuring tortuosity is difficult and is usually approximated by porosity. The diffusion constant was calculated and corrected for tortuosity as delineated in [2].

The unidimensionality assumption states that horizontal changes are minor relative to sediment changes with respect to depth. Unidimensionality was purported by Berner in [1] and is now commmonly accepted. The sediment-water interface can rise due to deposition of sediment or lower due to resuspension. The burial rate, $\omega$ is the rate of deposition minus resuspension. When there is no water flow, advection equals the burial rate. Assuming unidimensionality, the direction of the burial rate is determined by its sign. Assume $v$ is constant and is only due to burial, then $v=\omega$.

Hence, equation (2) becomes equation (3). When there is no change with respect to time, (3) becomes (4) $($ see $[1])$.

$$
\begin{gathered}
\frac{\partial C}{\partial t}=D \frac{\partial^{2} C}{\partial x^{2}}-\omega \frac{\partial C}{\partial x}+\sum R \\
0=D \frac{d^{2} C}{d x^{2}}-\omega \frac{d C}{d x}+\sum R
\end{gathered}
$$

Steady-state diagenesis may occur when the changes in a particular sediment layer are buried such that no change occurs relative to the sediment-water interface. Approximating diagenesis with (4) at a particular time stage is useful because (4) is linear and easy to solve.

\subsection{Mathematical Method 1}

Assume the soil samples are collected at depths from 0 to $h \mathrm{~cm}$. and are collected on $\mathrm{n}$ different dates. Then the steady-state diagenetic equation (4) models the phosphorus concentration. In addition, the burial of solid organic phosphorus, $\mathrm{P}$, and the bacterial decay of $\mathrm{P}$ into soluble phosphorus are given by

$$
-\omega \frac{d P}{d x}-\kappa P=0
$$

Solving (5) and applying the initial condition, $P(0)=P_{0}$, we obtain:

$$
P=P_{0} e^{-\frac{\kappa}{\omega} x} .
$$


Consequently, the linear model for phosphorus reaction used in this model is as follows:

$$
R=\kappa F P_{0} e^{-\frac{\kappa}{\omega} x}+\text { adsorption. }
$$

Thus, the linear representation for phosphorus concentration in this model is given by

$$
C^{\prime \prime}-\frac{(1+K) \omega}{D} C^{\prime}=-\frac{\kappa F P_{0}}{D} e^{\frac{\kappa}{\omega} x},
$$

where $\kappa$ is the rate constant for solid organic phosphorous decay, $F$ is the formation factor $\rho \frac{1-\phi}{\phi}, \phi$ total porosity and $\rho$ mean density, $K$ is the thermodynamic equilibrium constant, and $P_{0}$ is the solid labile organic phosphorous concentration.

The only unknown parameter in the diagenetic equation is $F P_{0}$. Though $P_{0}$ is not known, the upper bound for $P_{0}$ is known. The formation factor, $\mathrm{F}$, is unknown because the mean density, $\rho$ is unknown. Solving the diagenetic equation (8),

$$
C(x)=-\frac{\omega^{2} F P_{0}}{D \kappa+\omega(1+K)} e^{\frac{-\kappa}{\omega} x}+\kappa e^{\frac{(1+K) \omega}{D} x}+\zeta .
$$

Let

$$
\gamma=-\frac{\omega^{2} F P_{0}}{D \kappa+\omega(1+K)}, \quad \alpha=-\frac{\kappa}{\omega}, \quad \text { and } \quad \beta=\frac{(1+K) \omega}{D}
$$

then we get

$$
C(x)=\gamma e^{\alpha x}+\kappa e^{\beta x}+\zeta .
$$

In $(9), \gamma, \kappa$, and $\zeta$ are unknown parameters. For the first method, we determine the unknown parameters without boundary values using least square approximation. Assume that the samples $b=$ $\left(C_{x_{0} i}, C_{x_{1} i}, C_{x_{2} i}, \ldots C_{x_{m} i}\right)^{T}$ are collected at $t_{i}, i=0,1,2, \ldots n$ where $x_{k}, k=0,1,2, \ldots, m$, are depths with $x_{0}=0$. Let $A y=b$ where

$$
A=\left(\begin{array}{ccc}
1 & 1 & 1 \\
e^{x_{1} \alpha} & e^{x_{1} \beta} & 1 \\
e^{x_{2} \alpha} & e^{x_{2} \beta} & 1 \\
\vdots & \vdots & \vdots \\
e^{x_{m} \alpha} & e^{x_{m} \beta} & 1
\end{array}\right) \quad y=\left(\begin{array}{c}
\gamma_{i} \\
\kappa_{i} \\
\zeta_{i}
\end{array}\right) \quad \text { and } \quad b=\left(\begin{array}{c}
C_{x_{0} i} \\
C_{x_{1} i} \\
C_{x_{2} i} \\
\vdots \\
C_{x_{m} i}
\end{array}\right)
$$

at the fixed time, $t=t_{i}$.

If we solve $A^{T} A y=A^{T} b$ for $y=\left(\tilde{\gamma}_{i}, \tilde{\kappa}_{i}, \tilde{\zeta}_{i}\right)^{T}$, then the least square approximations of the unknown parameters $\gamma_{i}, \kappa_{i}$ and $\zeta_{i}$ are obtained at $t_{i}$.

Therefore, the mathematical method at $t_{i},(i=0,1,2, \ldots, n)$, is

$$
C\left(x, t_{i}\right)=\tilde{\gamma}_{i} e^{\alpha x}+\tilde{\kappa}_{i} e^{\beta x}+\tilde{\zeta}_{i} \quad 0 \leq x \leq h .
$$

(10) is an approximation to (9) with the given data where all parameters are known. Generally, $C\left(x_{j}, i\right)$ will not be equal to the sample data $C_{x_{i} j}(j=0, \ldots m, i=0, \ldots n)$.

\subsection{Mathematical Method 2}

We turn to the second method which comprises (9) and boundary conditions. Since $C_{x_{0} i}$, and $C_{x_{m} i}$ $(i=0, \ldots n)$ are given, we use these as boundary values with (9) to produce a second model that gives more accurate approximations.

Let

$$
\begin{aligned}
& \gamma_{i} e^{\alpha x_{0}}+\kappa_{i} e^{\beta x_{0}}+\zeta_{i}=C_{x_{0} i} \\
& \gamma_{i} e^{\alpha x_{m}}+\kappa_{i} e^{\beta x_{m}}+\zeta_{i}=C_{x_{m} i}
\end{aligned}
$$


Since $x_{0}=0$, one can get

$$
\begin{gathered}
\kappa_{i}=\frac{\left(C_{x_{0} i}-C_{x_{m} i}\right)-\gamma_{i}\left(1-e^{\alpha x_{m}}\right)}{\left(1-e^{\beta x_{m}}\right)} \\
=-\gamma_{i} \frac{\left(1-e^{\alpha x_{m}}\right)}{\left(1-e^{\beta x_{m}}\right)}+\frac{\left(C_{x_{0} i}-C_{x_{m} i}\right)}{\left(1-e^{\beta x_{m}}\right)} \\
\zeta_{i}=\frac{\left(C_{x_{0} i} e^{\beta x_{m}}-C_{x_{m} i}\right)-\gamma_{i}\left(e^{\beta x_{m}}-e^{\alpha x_{m}}\right)}{\left(e^{\beta x_{m}}-1\right)} \\
=-\gamma_{i} \frac{\left(e^{\beta x_{m}}-e^{\alpha x_{m}}\right)}{\left(e^{\beta x_{m}}-1\right)}+\frac{\left(C_{x_{0} i} e^{\beta x_{m}}-C_{x_{m} i}\right)}{\left(e^{\beta x_{m}}-1\right)} \\
i=0, \ldots n
\end{gathered}
$$

Substituting $\kappa_{i}$ and $\zeta_{i}$ in (9), one has the following:

$$
C(x)=\bar{\gamma}_{i} e^{\alpha x}+\bar{\kappa}_{i} e^{\beta x}+\bar{\zeta}_{i},
$$

where $\bar{\gamma}_{i}$ is still unknown since $\gamma_{i}$ is unknown,

$$
\overline{\kappa_{i}}=\frac{\left(C_{x_{0} i}-C_{x_{m} i}\right)}{\left(1-e^{\beta x_{m}}\right)}
$$

and

are known.

$$
\bar{\zeta}_{i}=\frac{\left(C_{x_{0} i} e^{\beta x m}-C_{x_{m} i}\right)}{\left(e^{\beta x m}-1\right)}
$$

To find approximation of $\bar{\gamma}_{i}$, the least quare approximation can be used. Let

$$
A=\left(\begin{array}{c}
e^{x_{1} \alpha} \\
e^{x_{2} \alpha} \\
\vdots \\
e^{x_{m-1} \alpha}
\end{array}\right) \quad y=\left(\gamma_{i}\right) \quad \text { and } \quad b=\left(\begin{array}{c}
C_{x_{1} i}-\kappa_{i} e^{\beta x_{1}}-\zeta_{i} \\
C_{x_{2} i}-\kappa_{i} e^{\beta x_{2}}-\zeta_{i} \\
\vdots \\
C_{x_{m-1} i}-\kappa_{i} e^{\beta x_{m-1}}-\zeta_{i}
\end{array}\right)
$$

at the fixed time, $t=t_{i}$.

Solving $A^{T} A y=A^{T} b$ for $y=\tilde{\gamma}_{i}$, we have $\tilde{\gamma}_{i}=\left(A^{T} b\right) /\left(A^{T} A\right)$. Now, we have

$$
C\left(x, t_{i}\right)=\tilde{\gamma}_{i} e^{\alpha x}+\tilde{\kappa}_{i} e^{\beta x}+\tilde{\zeta}_{i} \quad 0 \leq x \leq h .
$$

\section{Homopoty Continuation}

Formulas (10) and (11) provide approximations of phosphorus concentration $C\left(x, t_{i}\right)$ at any $x$ where $0 \leq x \leq h$ at a fixed time stage $t_{i}$. However, these do not provide any information for times between two time stages, a shortcoming of existing models. In reality, the concentration function $C(x, t)$ depends continuously on time (as well as on the position) and should provide estimates of concentrations between time stages. Since samples are taken only at some discrete time stages, the existing drawback may be overcome by use of smooth mappings that link the time stages. Homotopy continuation $[6,7]$ is one such mapping method. Hence, for $0 \leq x \leq h$, we construct a homotopy continuation as follows:

$$
C(x, t)= \begin{cases}\left(1-\frac{t}{t_{1}}\right) C\left(x, t_{0}\right)+\frac{t}{t_{1}} C\left(x, t_{1}\right), & t_{0} \leq t \leq t_{1} \\ \left(1-\frac{t-t_{1}}{t_{2}-t_{1}}\right) C\left(x, t_{1}\right)+\frac{t-t_{1}}{t_{2}-t_{1}} C\left(x, t_{2}\right), & t_{1} \leq t \leq t_{2} \\ \vdots & \vdots \\ \left(1-\frac{t-t_{n-1}}{t_{n}-t_{n-1}}\right) C\left(x, t_{n-1}\right)+\frac{t-t_{n-1}}{t_{n}-t_{n-1}} C\left(x, t_{n}\right), & t_{n-1} \leq t \leq t_{n}\end{cases}
$$


The method (12) provides approximations of the concentrations values $C(x, t)$, for $0 \leq x \leq h$ and $t_{0} \leq t \leq t_{n}$. If $x=x_{i}$ and $t=t_{j}$ for some $i$ and $j$, then the value $C(x, t)$ is one of the exact sample values (if $t_{j}$ is a time stage ) or an estimate using (12). Thus, the homotopy mapping continues approximations of the phosphorus concentrations at times between sample time stages.

We now present a numerical algorithm for calculating the concentrations between time stages. Suppose we wish to use the formula (10) to determine the value of

$C(\tilde{x}, \tilde{t})$, where $t_{i-1} \leq \tilde{t} \leq t_{i}$.

1. Input given values of $\tilde{x}, \tilde{t}, \alpha$ and $\beta$, and sample data matrix $C$, where $C_{i j}$ is the sample value at depth $x_{i}$ and time stage $t_{j},(i=0,1, \ldots m$ and $j=0,1, \ldots n)$.

2. Input $A$, where $a_{i 1}=e^{x_{i} \alpha}, a_{i 2}=e^{x_{i} \beta}$ and $a_{i 3}=1,(i=0,1, \ldots m)$.

3 .

do $\quad i=0, n$

$$
\begin{array}{ll}
\text { do } & j=0, m \\
& b_{j i}=C_{j i} \\
\text { solve } & A^{T} A y=A^{T} b_{j}, \text { for } y
\end{array}
$$

end do

do $\quad k=1,3$

end do

$$
z(i, k)=y(k)
$$

end do

4. $\quad C\left(\tilde{x}, t_{i-1}\right)=Z(i-1, k) b(\tilde{x})$, where $b(\tilde{x})=\left(e^{\alpha \tilde{x}}, e^{\beta \tilde{x}}, 1\right)^{T}$ and $k=1,2,3$.

5. $\quad C(\tilde{x}, \tilde{t})=\left(1-\frac{\tilde{t}-t_{i-1}}{t_{i}-t_{i-1}}\right) C\left(\tilde{x}, t_{i-1}\right)+\frac{\tilde{t}-t_{i-1}}{t_{i}-t_{i-1}} C\left(\tilde{x}, t_{i}\right)$

For the second method, the algorithm is similar.

\section{Example:}

Soil samples were collected at depths, 0.0, 0.25, 0.75, 1.5, and 3.0 centimeters on 4 different dates, days $0,30,73$, and 442 from a bay near the Gulf of Mexico [8]. Using the phosphorus concentration model (11), one could obtain the following:

$$
\begin{aligned}
& C(x, 0)=-4.462 e^{-0.005 x}-1.739 e^{0.011 x}+6.202 \\
& C(x, 30)=-11.028 e^{-0.005 x}-4.225 e^{0.011 x}+15.253 \\
& C(x, 73)=-23.910 e^{-0.005 x}-9.022 e^{0.011 x}+32.932 \\
& C(x, 442)=-0.405 e^{-0.005 x}-0.172 e^{0.011 x}+0.577 .
\end{aligned}
$$

Method (10) can be used similarly. These methods give approximations on day 0, 30, 73 and 442 at any depths from 0.0 to 3.0 centimeters, respectively. However, no approximation can be obtained at $(x, t)$ where $0.0 \leq x \leq 3.0$ and $0 \leq t \leq 442$. For the local citizens and legislatures, they may ask for the data at a particular date and depth, for example, at day 45 at depth 2.2 centimeters. Using method (12), one can obtain the following:

$$
C(x, t)= \begin{cases}\left(1-\frac{t}{30}\right) C(x, 0)+\frac{t}{30} C(x, 30), & 0 \leq t \leq 30 \\ \left(1-\frac{t-30}{43}\right) C(x, 30)+\frac{t-30}{43} C(x, 73), & 30 \leq t \leq 73 \\ \left(1-\frac{t-73}{369}\right) C(x, 73)+\frac{t-73}{369} C(x, 442), & 73 \leq t \leq 442\end{cases}
$$

The formula (14) clearly provides all approximations at $(x, t)$ where $0.0 \leq x \leq 3.0$ and $0 \leq t \leq 442$. For instance, $C(2.2,45)=0.0257$.

\section{Conclusion}

The approaches (10) and (11) are similar to established models that predict phosphorus concentrations at the specific sample time stages. These lack the ability to estimate concentration values at times between sample time stages. However, the preceding homotopy continuation scheme (12) empowers us to approximate concentration values between sample time stages. Thus, either method (10) or (11) combined 
with the method (12) is an improvement of the existing models that have been in use for decades since our combined methods give information about concentrations between time stages. With boundary conditions, (11) provides more accurate approximations than (10) under the homotopy framework. Moreover, the numerical algorithm that we have presented above provides a simple, user-friendly method for computing concentration values as shown in the example above. In order to improve the current proposed combined models, one could use more sample collection points as well as shortening the time between time stages. Although homotopy continuation will always bridge the gap between time stages, its accuracy decreases as the length of time gap increases.

\section{References}

1. B. Berner, Early Diagenesis, A Theoretical Approach, Princeton University Press, New Jersey, (1980).

2. B. Boudreau, Diagenetic Models and Their Implementation, New York, Springer-Verlag, (1997).

3. B. Boudreau, A Method-of-Lines Code for Carbon and Nutrient Diagenesis in Aquatic Sediments, Computers and Geosciences (1996), 22, 479-496.

4. Z. Ji, Hydrodynamics and Water Quality: Modeling Rivers, Lakes, and Estuaries, John Wiley \& Sons, New Jersey, (2008).

5. F. Meysman, et. al, Reactive Transport in Surface Sediments. I. Model Complexity and Software Quality, Computers and Geosciences (2003) 29, 91-300.

6. K. Li, T. Y. Li, An Algorithm for Symmetric Tridiagonal Eigenproblems —— Divide and Conquer with Homotopy Continuation, SIAM J. Sci. Comput. Vol.14, No. 3, (1993), 735-751.

7. K. Li, T. Y. Li, A Homotopy Algorithm for Symmetric Generalized Eigenproblem, Numerical Algorithms 4(1993), 167-195.

8. N. Davila, K. Li, Mathematical Models for Dissolved Phosphorus Concentrations in the Gulf of Mexico, Tech. Report, Department of Mathematics and Statistics, University of West Florida, May 2008. 